Available online at www.atnaa.org

Research Article

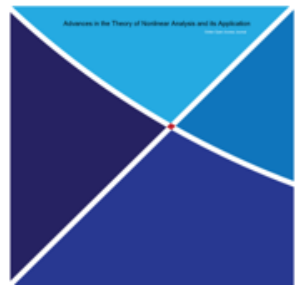

\title{
On the existence of solutions for a class of fourth order elliptic equations of Kirchhoff type with variable exponent
}

\author{
Nguyen Thanh Chung ${ }^{a}$
}

${ }^{a}$ Department of Mathematics, Quang Binh University, 312 Ly Thuong Kiet, Dong Hoi, Quang Binh, Vietnam

\section{Abstract}

In this paper, we consider a class of fourth order elliptic equations of Kirchhoff type with variable exponent

$$
\left\{\begin{array}{l}
\Delta_{p(x)}^{2} u-M\left(\int_{\Omega} \frac{1}{p(x)}|\nabla u|^{p(x)} d x\right) \Delta_{p(x)} u=\lambda f(x, u) \quad \text { in } \Omega, \\
u=\Delta u=0 \quad \text { on } \partial \Omega,
\end{array}\right.
$$

where $\Omega \subset \mathbb{R}^{N}, N \geq 3$, is a smooth bounded domain, $M(t)=a+b t^{\kappa}, a, \kappa>0, b \geq 0, \lambda$ is a positive parameter, $\Delta_{p(x)}^{2} u=\Delta\left(|\Delta u|^{p(x)-2} \Delta u\right)$ is the operator of fourth order called the $p(x)$-biharmonic operator, $\Delta_{p(x)} u=\operatorname{div}\left(|\nabla u|^{p(x)-2} \nabla u\right)$ is the $p(x)$-Laplacian, $p: \bar{\Omega} \rightarrow \mathbb{R}$ is a log-Hölder continuous function and $f: \bar{\Omega} \times \mathbb{R} \rightarrow \mathbb{R}$ is a continuous function satisfying some certain conditions. Using Ekeland's variational principle combined with variational techniques, an existence result is established in an appropriate function space.

Keywords: Fourth order elliptic equations; Kirchhoff type problems; Variable exponents; Ekeland's variational principle.

2010 MSC: 35J60, 35J35, 35G30, 46E35.

\section{Introduction}

In this paper, we consider a class of fourth order elliptic equations of Kirchhoff type with variable exponent

$$
\left\{\begin{array}{l}
\Delta_{p(x)}^{2} u-M\left(\int_{\Omega} \frac{1}{p(x)}|\nabla u|^{p(x)} d x\right) \Delta_{p(x)} u=\lambda f(x, u) \quad \text { in } \Omega \\
u=\Delta u=0 \quad \text { on } \partial \Omega
\end{array}\right.
$$

Email address: ntchung82@yahoo.com (Nguyen Thanh Chung) 
where $\Omega \subset \mathbb{R}^{N}, N \geq 3$, is a smooth bounded domain, $M(t)=a+b t^{\kappa}, a, \kappa>0, b \geq 0, \lambda$ is a positive parameter, $\Delta_{p(x)}^{2} u=\Delta\left(|\Delta u|^{p(x)-2} \Delta u\right)$ is the operator of fourth order called the $p(x)$-biharmonic operator, $\Delta_{p(x)} u=\operatorname{div}\left(|\nabla u|^{p(x)-2} \nabla u\right)$ is the $p(x)$-Laplacian, the exponent $p: \bar{\Omega} \rightarrow \mathbb{R}$ is log-Hölder continuous, that is, there exists $\bar{c}>0$ such that $|p(x)-p(y)| \leq-\frac{\bar{c}}{\log |x-y|}$ for all $x, y \in \bar{\Omega}$ with $0<|x-y| \leq \frac{1}{2}$ and $1<p^{-}:=\inf _{x \in \bar{\Omega}} p(x) \leq p^{+}:=\sup _{x \in \bar{\Omega}} p(x)<\frac{N}{2}, f: \bar{\Omega} \times \mathbb{R} \rightarrow \mathbb{R}$ is a continuous function.

We point out that if $p($.$) is a constant then problem (1) has been studied by many authors in recent$ years, we refer to some interesting papers [3, 8, 15, 19, 23, 27, 28, 29]. In [29], Wang and An considered the following fourth-order elliptic equation

$$
\left\{\begin{array}{l}
\Delta^{2} u-M\left(\int_{\Omega}|\nabla u|^{2} d x\right) \Delta u=f(x, u) \quad \text { in } \Omega \\
u=\Delta u=0 \text { on } \partial \Omega
\end{array}\right.
$$

where $\Omega \subset \mathbb{R}^{N}, N \geq 1$, is a smooth bounded domain, $f: \Omega \times \mathbb{R} \rightarrow \mathbb{R}$ and $M:[0,+\infty) \rightarrow \mathbb{R}$ are continuous functions. This problem is related to the stationary analog of the evolution equation of Kirchhoff type

$$
u_{t t}+\Delta^{2} u-M\left(\int_{\Omega}|\nabla u|^{2} d x\right) \Delta u=f(x, t),
$$

where $\Delta^{2}$ is the biharmonic operator, $\nabla u$ denotes the spatial gradient of $u$, see $[5]$ for the meaning of the problem from the point of view of physics and engineering. By assuming that $M$ is bounded on $[0,+\infty)$ and the nonlinear term $f$ satisfies the Ambrosetti-Rabinowitz type condition, Wang et al. obtained in 29. at least one nontrivial solution for problem (2) using the mountain pass theorem. Moreover, the authors also showed the existence at least two solutions in the case when $f$ is asymptotically linear at infinity. After that, Wang et al. 28] studied problem (2) in the case when $M$ is unbounded function, i.e. $M(t)=a+b t$, where $a>0, b \geq 0$ by using the mountain pass techniques and the truncation method. Some extensions regarding these results can be found in [3, 8, 15, 27] in which the authors considered problem $(2)$ in $\mathbb{R}^{N}$ or the nonlinearities involved critical exponents. In [19, 23], problem (1) has been studied in the general case when $p()=.p \in(1,+\infty)$ is a constant.

In recent years, the study of differential equations and variational problems with nonstandard $p(x)$ growth conditions has received more and more interest. The reason of such interest starts from the study of the role played by their applications in mathematical modelling of non-Newtonian fluids, in particular, the electrorheological fluids and of other phenomena related to image processing, elasticity and the flow in porous media, we refer the readers to [26, 31] for more details. Some results on problems involving $p(x)$-Laplace operator or $p(x)$-biharmonic operator can be found in [4, 6, 7, 9, 21, 22, 24. These types of operators where $p($.$) is a continuous function possess more complicated properties than the constant cases, mainly due to$ the fact that they are not homogeneous. We also find that Kirchhoff type problems with variable exponents has received a lot of attention in recent years, see for example [1, 2, 10, 13, 14, 20]. In [11, 12, we first studied the existence and multiplicity of solutions for elliptic problems of Kirchhoff type involving both $p(x)$-Laplace operator and $p(x)$-biharmonic operator in Sobolev spaces with variable exponents. Motivated by the contributions cited above, we shall study the existence of solutions for fourth order elliptic equations with variable exponents of the form (1). Our main tools come from Ekeland's variational principle combined with variational techniques in critical point theory. Actually, the results of this paper are natural extensions from those presented in [11, 12] and [20]. Moreover, we believe that our results introduced here are new even in the case when $p()=$.$p is a positive constant, see [28, 29], since the nonlinear term f$ is sublinear at infinity and it is allowed to change sign.

In order to study problem (1), let recall some definitions and basic properties of the generalized LebesgueSobolev spaces $L^{p(x)}(\Omega)$ and $W^{k, p(x)}(\Omega)$ where $\Omega$ is an open subset of $\mathbb{R}^{N}$. In that context, we refer to the books of Diening et al. [16] and Musielak [25], the papers of Ayoujil et al. 4], Boureanu et al. [7] and Zang et al [30]. Set

$$
C_{+}(\bar{\Omega}):=\{h ; h \in C(\bar{\Omega}), h(x)>1 \text { for all } x \in \bar{\Omega}\} .
$$


For any $h \in C_{+}(\bar{\Omega})$ we define

$$
h^{+}=\sup _{x \in \bar{\Omega}} h(x) \text { and } h^{-}=\inf _{x \in \bar{\Omega}} h(x) .
$$

For any $p(x) \in C_{+}(\bar{\Omega})$, we define the variable exponent Lebesgue space

$$
L^{p(x)}(\Omega)=\left\{u: \text { a measurable real-valued function such that } \int_{\Omega}|u(x)|^{p(x)} d x<\infty\right\} .
$$

We recall the following so-called Luxemburg norm on this space defined by the formula

$$
|u|_{p(x)}=\inf \left\{\mu>0: \quad \int_{\Omega}\left|\frac{u(x)}{\mu}\right|^{p(x)} d x \leq 1\right\} .
$$

Variable exponent Lebesgue spaces resemble classical Lebesgue spaces in many respects: they are Banach spaces, the Hölder inequality holds, they are reflexive if and only if $1<p^{-} \leq p^{+}<\infty$ and continuous functions are dense if $p^{+}<\infty$. The inclusion between Lebesgue spaces also generalizes naturally: if $0<$ $|\Omega|<\infty$ and $p_{1}, p_{2}$ are variable exponents so that $p_{1}(x) \leq p_{2}(x)$ a.e. $x \in \Omega$ then there exists the continuous embedding $L^{p_{2}(x)}(\Omega) \hookrightarrow L^{p_{1}(x)}(\Omega)$. We denote by $L^{p^{\prime}(x)}(\Omega)$ the conjugate space of $L^{p(x)}(\Omega)$, where $\frac{1}{p(x)}+$ $\frac{1}{p^{\prime}(x)}=1$. For any $u \in L^{p(x)}(\Omega)$ and $v \in L^{p^{\prime}(x)}(\Omega)$ the Hölder inequality

$$
\left|\int_{\Omega} u v d x\right| \leq\left(\frac{1}{p^{-}}+\frac{1}{\left(p^{\prime}\right)^{-}}\right)|u|_{p(x)}|v|_{p^{\prime}(x)}
$$

holds true.

An important role in manipulating the generalized Lebesgue-Sobolev spaces is played by the modular of the $L^{p(x)}(\Omega)$ space, which is the mapping $\rho_{p(x)}: L^{p(x)}(\Omega) \rightarrow \mathbb{R}$ defined by

$$
\rho_{p(x)}(u)=\int_{\Omega}|u|^{p(x)} d x .
$$

If $u \in L^{p(x)}(\Omega)$ and $p^{+}<\infty$ then the following relations hold

$$
|u|_{p(x)}^{p^{-}} \leq \rho_{p(x)}(u) \leq|u|_{p(x)}^{p^{+}}
$$

provided $|u|_{p(x)}>1$ while

$$
|u|_{p(x)}^{p^{+}} \leq \rho_{p(x)}(u) \leq|u|_{p(x)}^{p^{-}}
$$

provided $|u|_{p(x)}<1$ and

$$
\left|u_{n}-u\right|_{p(x)} \rightarrow 0 \Leftrightarrow \rho_{p(x)}\left(u_{n}-u\right) \rightarrow 0 .
$$

As in the constant case, for any positive integer $k$, the Sobolev space with variable exponent $W^{k, p(x)}(\Omega)$ is defined by

$$
W^{k, p(x)}(\Omega)=\left\{u \in L^{p(x)}(\Omega): D^{\alpha} u \in L^{p(x)}(\Omega),|\alpha| \leq k\right\},
$$

where $D^{\alpha} u=\frac{\partial^{|\alpha|}}{\partial x_{1}^{\alpha_{1}} \partial x_{2}^{\alpha_{2}} \ldots \partial x_{N}^{\alpha_{N}}} u$, with $\alpha=\left(\alpha_{1}, \ldots, \alpha_{N}\right)$ is a multi-index and $|\alpha|=\sum_{i=1}^{N} \alpha_{i}$. The space $W^{k, p(x)}(\Omega)$ equipped with the norm

$$
\|u\|_{k, p(x)}=\sum_{|\alpha| \leq k}\left|D^{\alpha} u\right|_{p(x)},
$$

also becomes a separable and reflexive Banach space. Due to the log-Hölder continuity of the exponent $p$, the space $C^{\infty}(\Omega)$ is dense in $W^{k, p(x)}(\Omega)$. Moreover, we have the following embedding results. 
Proposition 1.1 (see [4]). For $p, r \in C_{+}(\bar{\Omega})$ such that $r(x) \leq p_{k}^{*}(x)$ for all $x \in \bar{\Omega}$, there is a continuous embedding

$$
W^{k, p(x)}(\Omega) \hookrightarrow L^{r(x)}(\Omega),
$$

where $p_{k}^{*}(x)=\frac{N p(x)}{N-k p(x)}$ if $k p(x)<N$ and $p_{k}^{*}(x)=+\infty$ if $k p(x)>N$. If we replace $\leq$ with $<$, the embedding is compact.

We denote by $W_{0}^{k, p(x)}(\Omega)$ the closure of $C_{0}^{\infty}(\Omega)$ in $W^{k, p(x)}(\Omega)$. Note that the weak solutions of problem (1) are considered in the generalized Sobolev space

$$
X=W_{0}^{1, p(x)}(\Omega) \cap W^{2, p(x)}(\Omega)
$$

equipped with the norm $\|u\|_{X}=\|u\|_{1, p(x)}+\|u\|_{2, p(x)}$ or $\|u\|_{X}=|u|_{p(x)}+|\nabla u|_{p(x)}+\sum_{\alpha=2}\left|D^{\alpha} u\right|_{p(x)}$.

According to [30], the norm $\|\cdot\|_{X}$ is equivalent to the norm $|\Delta .|_{p(x)}$ in the space $X$. Consequently, the norms $\|\cdot\|_{2, p(x)},\|\cdot\|_{X}$ and $|\Delta \cdot|_{p(x)}$ are equivalent. For this reason, we can consider in the space $X$ the following equivalent norms

$$
\|u\|=|\Delta u|_{p(x)}+|\nabla u|_{p(x)}
$$

and

$$
\|u\|=\inf \left\{\mu>0: \quad \int_{\Omega}\left(\left|\frac{\Delta u(x)}{\mu}\right|^{p(x)}+\left|\frac{\nabla u(x)}{\mu}\right|^{p(x)}\right) d x \leq 1\right\} .
$$

Let us define the functional $\Lambda: X \rightarrow \mathbb{R}$ by

$$
\Lambda(u)=\int_{\Omega}\left(|\Delta u|^{p(x)}+|\nabla u|^{p(x)}\right) d x, u \in X,
$$

then using similar arguments as in [7, Proposition 1] we obtain the following modular-type inequalities.

Proposition 1.2. For $u, u_{n} \in X$ and the functional $\Lambda: X \rightarrow \mathbb{R}$ define as in (12), we have the following assertions:

(1) $\|u\|<1$ (respectively $=1 ;>1) \Longleftrightarrow \Lambda(u)<1$ (respectively $=1 ;>1$ );

(2) $\|u\| \leq 1 \Rightarrow\|u\|^{p^{+}} \leq \Lambda(u) \leq\|u\|^{p^{-}}$;

(3) $\|u\| \geq 1 \Rightarrow\|u\|^{p^{-}} \leq \Lambda(u) \leq\|u\|^{p^{+}}$;

(4) $\left\|u_{n}\right\| \rightarrow 0$ (respectively $\left.\rightarrow \infty\right) \Longleftrightarrow \Lambda\left(u_{n}\right) \rightarrow 0$ (respectively $\rightarrow \infty$ ) as $n \rightarrow \infty$.

\section{Main results}

In this section, we discuss the existence of nontrivial weak solutions of problem (1). Let us denote by $c_{i}, i=1,2, \ldots$ general positive constants whose value may change from line to line. We shall look for weak solutions of problem (1) in the space $X:=W_{0}^{1, p(x)}(\Omega) \cap W^{2, p(x)}(\Omega)$ with the norm mentioned as in Section 1.

For the function $f: \bar{\Omega} \times \mathbb{R} \rightarrow \mathbb{R}$ mentioned above, let us set $F(x, t)=\int_{0}^{t} f(x, s) d s$. Throughout this section, we assume that

$\left(\mathcal{H}_{1}\right) F: \bar{\Omega} \times \mathbb{R} \rightarrow \mathbb{R}$ is a $C^{1}$ function such that

$$
F(x, \tau t)=\tau^{q(x)} F(x, t), \text { for all } \tau>0, \quad x \in \bar{\Omega}, \quad t \in \mathbb{R}
$$

where $q \in C_{+}(\bar{\Omega})$;

$\left(\mathcal{H}_{2}\right)$ There exists $\Omega_{0} \subset \subset \Omega$ with meas $\left(\Omega_{0}\right)>0$ such that $F(x, t)>0$ for all $x \in \Omega_{0}$ and all $t \in \mathbb{R}$; 
$\left(\mathcal{H}_{3}\right) 1<q^{+}<p^{-} \leq p^{+}<p_{2}^{*}(x)=\frac{N p(x)}{N-2 p(x)}$ for all $x \in \bar{\Omega}$.

Remark 2.1. From assumption $\left(\mathcal{H}_{1}\right)$, for all $x \in \bar{\Omega}$ and $t \in \mathbb{R}$, we have the so-called Euler indentity

$$
f(x, t) t=q(x) F(x, t)
$$

and

$$
|F(x, t)| \leq C|t|^{q(x)} \text { and }|f(x, t)| \leq C|t|^{q(x)-1}, \quad \forall x \in \bar{\Omega}, t \in \mathbb{R}
$$

for some positive constant $C$.

Definition 2.2. We say that $u \in X$ is a weak solution of problem (1) if

$$
\begin{gathered}
\int_{\Omega}|\Delta u|^{p(x)-2} \Delta u \Delta v d x+\left[a+b\left(\int_{\Omega} \frac{1}{p(x)}|\nabla u|^{p(x)} d x\right)^{\kappa}\right] \int_{\Omega}|\nabla u|^{p(x)-2} \nabla u \cdot \nabla v d x \\
-\lambda \int_{\Omega} f(x, u) v d x=0
\end{gathered}
$$

for all $v \in X$.

For each $\lambda \in \mathbb{R}$, let us define the functional $J_{\lambda}: X \rightarrow \mathbb{R}$ by

$$
\begin{gathered}
J_{\lambda}(u)=\int_{\Omega} \frac{1}{p(x)}|\Delta u|^{p(x)} d x+a \int_{\Omega} \frac{1}{p(x)}|\nabla u|^{p(x)} d x+\frac{b}{\kappa+1}\left(\int_{\Omega} \frac{1}{p(x)}|\nabla u|^{p(x)} d x\right)^{\kappa+1} \\
-\lambda \int_{\Omega} F(x, u) d x, \quad u \in X
\end{gathered}
$$

we then deduce that $J_{\lambda} \in C^{1}(X, \mathbb{R})$ and its derivative is given by the formula

$$
\begin{aligned}
J_{\lambda}^{\prime}(u)(v)=\int_{\Omega}|\Delta u|^{p(x)-2} \Delta u \Delta v d x+ & {\left[a+b\left(\int_{\Omega} \frac{1}{p(x)}|\nabla u|^{p(x)} d x\right)^{\kappa}\right] \int_{\Omega}|\nabla u|^{p(x)-2} \nabla u \cdot \nabla v d x } \\
& -\lambda \int_{\Omega} f(x, u) v d x=0
\end{aligned}
$$

for all $u, v \in X$. Thus, we will seek weak solutions of problem (1) as the critical points of the functional $J_{\lambda}$. We first have the following lemma.

Lemma 2.3. Assume that conditions $\left(\mathcal{H}_{1}\right)-\left(\mathcal{H}_{3}\right)$ hold. Then, there exist constants $\alpha>0, \rho \in(0,1)$ and $\lambda^{*}>0$ such that, for any $\lambda \in\left(0, \lambda^{*}\right)$, we have $J_{\lambda}(u) \geq \alpha>0$ for all $u \in X$ with $\|u\|=\rho$.

Proof. Let $\rho \in(0,1)$ and $u \in X$ be such that $\|u\|=\rho$. Then we deduce from Remark 2.1 and the continuous embeddings $X \hookrightarrow L^{q^{ \pm}}(\Omega)$ that

$$
\int_{\Omega} F(x, u) d x \leq \int_{\Omega} C|u|^{q(x)} d x \leq c_{1}\left(\|u\|^{q^{-}}+\|u\|^{q^{+}}\right) .
$$

Hence,

$$
\begin{aligned}
J_{\lambda}(u)= & \int_{\Omega} \frac{1}{p(x)}|\Delta u|^{p(x)} d x+a \int_{\Omega} \frac{1}{p(x)}|\nabla u|^{p(x)} d x+\frac{b}{\kappa+1}\left(\int_{\Omega} \frac{1}{p(x)}|\nabla u|^{p(x)} d x\right)^{\kappa+1} \\
& -\lambda \int_{\Omega} F(x, u) d x \\
\geq & \frac{\min \{1, a\}}{p^{+}}\|u\|^{p^{+}}-\lambda c_{1}\left(\|u\|^{q^{-}}+\|u\|^{q^{+}}\right) \\
= & \left(\frac{\min \{1, a\}}{p^{+}}\|u\|^{p^{+}-q^{-}}-\lambda c_{1}\right)\|u\|^{q^{-}} .
\end{aligned}
$$


Set

$$
\lambda^{*}=\frac{\min \{1, a\}}{2 c_{1} p^{+}} \rho^{p^{+}-q^{-}}, \quad \alpha=\lambda^{*} \rho^{q^{-}},
$$

which yields for any $\lambda \in\left(0, \lambda^{*}\right)$ we have $J_{\lambda}(u) \geq \alpha>0$ for all $u \in X$ with $\|u\|=\rho$. The proof of Lemma 2.3 is complete.

Lemma 2.4. Assume that conditions $\left(\mathcal{H}_{1}\right)-\left(\mathcal{H}_{3}\right)$ hold. Then, there exists a function $u_{0} \in X \backslash\{0\}$ and $u_{0} \geq 0$ such that, $J_{\lambda}\left(t u_{0}\right)<0$ for all $t>0$ small enough.

Proof. Set $q_{0}^{-}:=\inf _{x \in \bar{\Omega}_{0}} q(x)$ and $p_{0}^{-}:=\inf _{x \in \bar{\Omega}_{0}} p(x)$. From condition $\left(\mathcal{H}_{3}\right)$ we have $q_{0}^{-}<p_{0}^{-}$, let $\epsilon_{0}>0$ be such that $q_{0}^{-}+\epsilon_{0}<p_{0}^{-}$. Since $q \in C\left(\bar{\Omega}_{0}\right)$, there exists an open set $\Omega_{1} \subset \subset \Omega_{0}$ such that $\left|q(x)-p_{0}^{-}\right|<\epsilon_{0}$ for all $x \in \Omega_{1}$. Thus,

$$
q(x) \leq q_{0}^{-}+\epsilon_{0}<p_{0}^{-}, \quad \forall x \in \Omega_{1} .
$$

Let $u_{0} \in C_{0}^{\infty}$ be such that $\operatorname{supp}\left(u_{0}\right) \subset \Omega_{1} \subset \subset \Omega_{0}, u_{0}=1$ in a subset $\Omega_{1}^{\prime} \subset \operatorname{supp}\left(u_{0}\right), 0 \leq u_{0} \leq 1$ in $\Omega_{1}$. Without loss of generality, we may assume that $\left\|u_{0}\right\|=1$, that is,

$$
\int_{\Omega_{0}}\left|\Delta u_{0}\right|^{p(x)} d x+\int_{\Omega_{0}}\left|\nabla u_{0}\right|^{p(x)} d x=1
$$

Hence, by condition $\left(\mathcal{H}_{1}\right)$, for any $t \in(0,1)$ we obtain

$$
\begin{aligned}
J_{\lambda}\left(t u_{0}\right)= & \int_{\Omega} \frac{1}{p(x)}\left|\Delta\left(t u_{0}\right)\right|^{p(x)} d x+a \int_{\Omega} \frac{1}{p(x)}\left|\nabla\left(t u_{0}\right)\right|^{p(x)} d x+\frac{b}{\kappa+1}\left(\int_{\Omega} \frac{1}{p(x)}\left|\nabla\left(t u_{0}\right)\right|^{p(x)} d x\right)^{\kappa+1} \\
& -\lambda \int_{\Omega} F\left(x, t u_{0}\right) d x \\
\leq & \frac{t^{p_{0}^{-}}}{p_{0}^{-}} \int_{\Omega_{0}}\left|\Delta u_{0}\right|^{p(x)} d x+\frac{a t^{p_{0}^{-}}}{p_{0}^{-}} \int_{\Omega_{0}}\left|\nabla u_{0}\right|^{p(x)} d x+\frac{b t^{p_{0}^{-}(\kappa+1)}}{\left(p_{0}^{-}\right)^{\kappa+1}(\kappa+1)}\left(\int_{\Omega_{0}}\left|\nabla u_{0}\right|^{p(x)} d x\right)^{\kappa+1} \\
& -\lambda \int_{\Omega_{1}} t^{q(x)} F\left(x, u_{0}\right) d x \\
\leq t^{p_{0}^{-}} & {\left[\int_{\Omega_{0}}\left|\Delta u_{0}\right|^{p(x)} d x+a \int_{\Omega_{0}}\left|\nabla u_{0}\right|^{p(x)} d x+\frac{b}{\kappa+1}\left(\int_{\Omega_{0}}\left|\nabla u_{0}\right|^{p(x)} d x\right)^{\kappa+1}\right] } \\
& -\lambda t^{q_{0}^{-}+\epsilon_{0}} \int_{\Omega_{1}} F\left(x, u_{0}\right) d x .
\end{aligned}
$$

By condition $\left(\mathcal{H}_{2}\right)$, we have $\int_{\Omega_{1}} F\left(x, u_{0}\right) d x>0$ and thus, $J_{\lambda}\left(t u_{0}\right)<0$ for all $0<t<\delta^{\frac{1}{p_{0}^{-}-q_{0}^{-}-\epsilon_{0}}}$ with $0<\delta<\min \left\{1, \delta_{0}\right\}$ and

$$
\delta_{0}:=\frac{\lambda \int_{\Omega_{1}} F\left(x, u_{0}\right) d x}{\int_{\Omega_{0}}\left|\Delta u_{0}\right|^{p(x)} d x+a \int_{\Omega_{0}}\left|\nabla u_{0}\right|^{p(x)} d x+\frac{b}{\kappa+1}\left(\int_{\Omega_{0}}\left|\nabla u_{0}\right|^{p(x)} d x\right)^{\kappa+1}} .
$$

Note that by (13),

$$
\int_{\Omega_{0}}\left|\Delta u_{0}\right|^{p(x)} d x+a \int_{\Omega_{0}}\left|\nabla u_{0}\right|^{p(x)} d x+\frac{b}{\kappa+1}\left(\int_{\Omega_{0}}\left|\nabla u_{0}\right|^{p(x)} d x\right)^{\kappa+1}>0
$$

and the proof of Lemma 2.4 is complete.

The main result of this paper can be stated as follows. 
Theorem 2.5. Assume that the conditions $\left(\mathcal{H}_{1}\right)-\left(\mathcal{H}_{3}\right)$ hold. Then there exists a constant $\lambda^{*}>0$ such that for any $\lambda \in\left(0, \lambda^{*}\right)$, problem (1) has a nontrivial weak solution.

Proof of Theorem 2.5. Let $\lambda^{*}>0$ be defined as in (11) and $\lambda \in\left(0, \lambda^{*}\right)$. By Lemma 2.3 it follows that on the boundary of the ball centered at the origin and of radius $\rho$ in $X$, denoted by $B_{\rho}(0)$, we have

$$
\inf _{\partial B_{\rho}(0)} J_{\lambda}>0 .
$$

On the other hand, by Lemma 2.4 there exists $u_{0} \in X$ such that $J_{\lambda}\left(t u_{0}\right)<0$ for all $t>0$ small enough. Moreover, by the proof of Lemma 2.3 we deduce that for any $u \in B_{\rho}(0)$,

$$
J_{\lambda}(u) \geq \frac{\min \{1, a\}}{p^{+}}\|u\|^{p^{+}}-\lambda c_{1}\|u\|^{q^{-}} .
$$

It follows that

$$
-\infty<\underline{c}:=\frac{\inf }{B_{\rho}(0)} J_{\lambda}<0 .
$$

Let $0<\epsilon<\inf _{\partial B_{\rho}(0)} J_{\lambda}-\inf _{B_{\rho}(0)} J_{\lambda}$. Using the above information, the functional $J_{\lambda}: \overline{B_{\rho}(0)} \longrightarrow \mathbb{R}$, is lower bounded on $\overline{B_{\rho}(0)}$ and $J_{\lambda} \in C^{1}\left(\overline{B_{\rho}(0)}, \mathbb{R}\right)$. Then by Ekeland's variational principle [18], there exists $u_{\epsilon} \in \overline{B_{\rho}(0)}$ such that

$$
\left\{\begin{array}{l}
\underline{c} \leq J_{\lambda}\left(u_{\epsilon}\right) \leq \underline{c}+\epsilon \\
0<J_{\lambda}(u)-J_{\lambda}\left(u_{\epsilon}\right)+\epsilon\left\|u-u_{\epsilon}\right\|, \quad u \neq u_{\epsilon}
\end{array}\right.
$$

Since

$$
J_{\lambda}\left(u_{\epsilon}\right) \leq \inf _{B_{\rho}(0)} J_{\lambda}+\epsilon \leq \inf _{B_{\rho}(0)} J_{\lambda}+\epsilon<\inf _{\partial B_{\rho}(0)} J_{\lambda},
$$

we deduce that $u_{\epsilon} \in B_{\rho}(0)$. Now, we define $\bar{J}_{\lambda}: \overline{B_{\rho}(0)} \longrightarrow \mathbb{R}$ by $\bar{J}_{\lambda}(u)=J_{\lambda}(u)+\epsilon\left\|u-u_{\epsilon}\right\|$. It is clear that $u_{\epsilon}$ is a minimum point of $\bar{J}_{\lambda}$ and thus

$$
\frac{\bar{J}_{\lambda}\left(u_{\epsilon}+t \cdot v\right)-\bar{J}_{\lambda}\left(u_{\epsilon}\right)}{t} \geq 0
$$

for small $t>0$ and any $v \in B_{1}(0)$. The above relation yields

$$
\frac{J_{\lambda}\left(u_{\epsilon}+t \cdot v\right)-J_{\lambda}\left(u_{\epsilon}\right)}{t}+\epsilon\|v\| \geq 0 .
$$

Letting $t \rightarrow 0$ it follows that $J_{\lambda}^{\prime}\left(u_{\epsilon}\right)(v)+\epsilon\|v\| \geq 0$ and we infer that $\left\|J_{\lambda}^{\prime}\left(u_{\epsilon}\right)\right\| \leq \epsilon$. Hence, there exists a sequence $\left\{u_{n}\right\} \subset B_{\rho}(0)$ satisfying

$$
J_{\lambda}\left(u_{n}\right) \rightarrow \underline{c}<0 \quad \text { and } \quad J_{\lambda}^{\prime}\left(u_{n}\right) \rightarrow 0_{X^{*}} .
$$

It is clear that $\left\{u_{n}\right\}$ is bounded in $X$. Thus, there exists $u$ in $X$ such that, up to a subsequence, $\left\{u_{n}\right\}$ converges weakly to $u$ in $X$. Since $q(x)<p_{2}^{*}(x)$ for all $x \in \bar{\Omega}$, we deduce that there exists a compact embedding $X \hookrightarrow L^{q(x)}(\Omega)$, hence the sequence $\left\{u_{n}\right\}$ converges strongly to $u$ in $L^{q(x)}(\Omega)$. Moreover, by 15 we deduce that $J_{\lambda}^{\prime}\left(u_{n}\right)\left(u_{n}-u\right) \rightarrow 0$ as $n \rightarrow \infty$. We also have $J_{\lambda}^{\prime}(u)\left(u_{n}-u\right) \rightarrow 0$ as $n \rightarrow \infty$ because $\left\{u_{n}\right\}$ converges weakly to $u$ in $X$, and thus,

$$
\lim _{n \rightarrow \infty}\left(J_{\lambda}^{\prime}\left(u_{n}\right)-J_{\lambda}^{\prime}(u)\right)\left(u_{n}-u\right)=0 .
$$


Using Hölder's inequality (4), Remark 2.1 and the compact embedding $X \hookrightarrow L^{q(x)}(\Omega)$ we have

$$
\begin{aligned}
\int_{\Omega}\left(f\left(x, u_{n}\right)-f(x, u)\right)\left(u_{n}-u\right) d x & \leq \int_{\Omega}\left(\left|u_{n}\right|^{q(x)-1}+|u|^{q(x)-1}\right)\left|u_{n}-u\right| d x \\
& \leq 2\left(\left.\left.|| u_{n}\right|^{q(x)-1}\right|_{\frac{q(x)}{q(x)-1}}+\left.\left.|| u\right|^{q(x)-1}\right|_{\frac{q(x)}{q(x)-1}}\right)\left|u_{n}-u\right|_{q(x)} \\
& \rightarrow 0, \quad n \rightarrow \infty,
\end{aligned}
$$

which implies that

$$
\lim _{n \rightarrow \infty} \int_{\Omega}\left(f\left(x, u_{n}\right)-f(x, u)\right)\left(u_{n}-u\right) d x=0 .
$$

Since the sequence $\left\{u_{n}\right\}$ converges weakly to $u$ in $X=W_{0}^{1, p(x)}(\Omega) \cap W^{2, p(x)}(\Omega)$, it is bounded and converges weakly to $u$ in $W_{0}^{1, p(x)}(\Omega)$, so we get

$$
\lim _{n \rightarrow \infty}\left(\int_{\Omega} \frac{1}{p(x)}\left|\nabla u_{n}\right|^{p(x)} d x\right)^{\kappa} \int_{\Omega}|\nabla u|^{p(x)-2} \nabla u \cdot\left(\nabla u_{n}-\nabla u\right) d x=0 .
$$

and

$$
\lim _{n \rightarrow \infty}\left(\int_{\Omega} \frac{1}{p(x)}|\nabla u|^{p(x)} d x\right)^{\kappa} \int_{\Omega}|\nabla u|^{p(x)-2} \nabla u \cdot\left(\nabla u_{n}-\nabla u\right) d x=0 .
$$

Let us recall the following elementary inequalities (see [4])

$$
\begin{gathered}
\left(|\xi|^{s-2} \xi-|\zeta|^{s-2} \zeta\right)(\xi-\zeta) \geq \frac{1}{2^{s}}|\xi-\zeta|^{s}, \quad s \geq 2, \\
\left(|\xi|^{s-2} \xi-|\zeta|^{s-2} \zeta\right)(\xi-\zeta)(|\xi|+|\zeta|)^{2-s} \geq(s-1)|\xi-\zeta|^{2}, \quad 1<s<2
\end{gathered}
$$

for all $\xi, \zeta \in \mathbb{R}^{N}$. Put

$$
U_{p(x)}:=\{x \in \Omega: p(x) \geq 2\}, \quad V_{p(x)}:=\{x \in \Omega: 1<p(x)<2\},
$$

then, it follows from 20 and 22 that

$$
\begin{gathered}
\int_{U_{p(x)}}\left|\Delta u_{n}-\Delta u\right|^{p(x)} d x \leq c_{2} \int_{\Omega} A^{(1)}\left(\Delta u_{n}, \Delta u\right) d x \\
\int_{U_{p(x)}}\left|\nabla u_{n}-\nabla u\right|^{p(x)} d x \leq c_{2} \int_{\Omega} A^{(N)}\left(\nabla u_{n}, \nabla u\right) d x \\
\int_{V_{p(x)}}\left|\Delta u_{n}-\Delta u\right|^{p(x)} d x \leq c_{3} \int_{\Omega}\left(A^{(1)}\left(\Delta u_{n}, \Delta u\right)\right)^{\frac{p(x)}{2}}\left(C^{(1)}\left(\Delta u_{n}, \Delta u\right)\right)^{(2-p(x)) \frac{p(x)}{2}} d x \\
\int_{V_{p(x)}}\left|\nabla u_{n}-\nabla u\right|^{p(x)} d x \leq c_{3} \int_{\Omega}\left(A^{(N)}\left(\nabla u_{n}, \nabla u\right)\right)^{\frac{p(x)}{2}}\left(C^{(N)}\left(\nabla u_{n}, \nabla u\right)\right)^{(2-p(x)) \frac{p(x)}{2}} d x,
\end{gathered}
$$

where $A^{(i)}, C^{(i)}: \mathbb{R}^{i} \times \mathbb{R}^{i} \rightarrow \mathbb{R}, i=1, N$ are defined by the following formulas

$$
A^{(i)}(\xi, \zeta):=\left(|\xi|^{p(x)-2} \xi-|\zeta|^{p(x)-2} \zeta\right)(\xi-\zeta), C^{(i)}(\xi, \zeta):=|\xi|+|\zeta|
$$

for all $\xi, \zeta \in \mathbb{R}^{i}, i=1, N$. 
Now, from relations (16)-19), we get

$$
\begin{aligned}
0 \leq \int_{\Omega} & \left(\left|\Delta u_{n}\right|^{p(x)-2} \Delta u_{n}-\left|\Delta u_{n}\right|^{p(x)-2} \Delta u_{n}\right)\left(\Delta u_{n}-\Delta u\right) d x \\
& +a \int_{\Omega}\left(\left|\nabla u_{n}\right|^{p(x)-2} \nabla u_{n}-\left|\nabla u_{n}\right|^{p(x)-2} \nabla u_{n}\right) \cdot\left(\nabla u_{n}-\nabla u\right) d x \\
& +b\left(\int_{\Omega} \frac{1}{p(x)}\left|\nabla u_{n}\right|^{p(x)} d x\right)^{\kappa} \int_{\Omega}\left(\left|\nabla u_{n}\right|^{p(x)-2} \nabla u_{n}-\left|\nabla u_{n}\right|^{p(x)-2} \nabla u_{n}\right) \cdot\left(\nabla u_{n}-\nabla u\right) d x \\
=\left(J_{\lambda}^{\prime}\left(u_{n}\right)-J_{\lambda}^{\prime}(u)\right)\left(u_{n}-u\right)+\lambda \int_{\Omega}\left(f\left(x, u_{n}\right)-f(x, u)\right)\left(u_{n}-u\right) d x & \\
& +b\left(\int_{\Omega} \frac{1}{p(x)}|\nabla u|^{p(x)} d x\right)^{\kappa} \int_{\Omega}|\nabla u|^{p(x)-2} \nabla u \cdot\left(\nabla u_{n}-\nabla u\right) d x \\
& \quad-b\left(\int_{\Omega} \frac{1}{p(x)}\left|\nabla u_{n}\right|^{p(x)} d x\right)^{\kappa} \int_{\Omega}\left|\nabla u_{n}\right|^{p(x)-2} \nabla u \cdot\left(\nabla u_{n}-\nabla u\right) d x \\
\rightarrow 0, & n \rightarrow \infty .
\end{aligned}
$$

Therefore, we have

$$
\lim _{n \rightarrow \infty} \int_{\Omega} A^{(1)}\left(\Delta u_{n}, \Delta u\right) d x=\lim _{n \rightarrow \infty} \int_{\Omega} A^{(N)}\left(\nabla u_{n}, \nabla u\right) d x=0 .
$$

For this reason, we can assume that $0 \leq \int_{\Omega} A^{(1)}\left(\Delta u_{n}, \Delta u\right) d x<1$. If $\int_{\Omega} A^{(1)}\left(\Delta u_{n}, \Delta u\right) d x=0$ then $A^{(1)}\left(\Delta u_{n}, \Delta u\right)=0$ since $A^{(1)}\left(\Delta u_{n}, \Delta u\right) \geq 0$ in $\Omega$. If $0<\int_{\Omega} A^{(1)}\left(\Delta u_{n}, \Delta u\right) d x<1$, then thanks to the Young inequality

$$
a b \leq \frac{a^{r}}{r}+\frac{b^{r^{\prime}}}{r^{\prime}}, \quad \forall a, b>0, \quad \frac{1}{r}+\frac{1}{r^{\prime}}=1, \quad r, r^{\prime} \in(1,+\infty)
$$

with

$$
\begin{gathered}
a=\left(A^{(1)}\left(\Delta u_{n}, \Delta u\right)\right)^{\frac{p(x)}{2}}\left(\int_{V_{p(x)}} A^{(1)}\left(\Delta u_{n}, \Delta u\right) d x\right)^{\frac{-p(x)}{2}}, \quad b=\left(C^{(1)}\left(\Delta u_{n}, \Delta u\right)\right)^{(2-p(x)) \frac{p(x)}{2}}, \\
r=\frac{2}{p(x)}, \quad r^{\prime}=\frac{2}{2-p(x)}, \quad x \in V_{p(x)},
\end{gathered}
$$

we conclude that

$$
\begin{aligned}
& \left(\int_{V_{p(x)}} A^{(1)}\left(\Delta u_{n}, \Delta u\right) d x\right)^{-\frac{1}{2}} \int_{V_{p(x)}}\left(A^{(1)}\left(\Delta u_{n}, \Delta u\right)\right)^{\frac{p(x)}{2}}\left(C^{(1)}\left(\Delta u_{n}, \Delta u\right)\right)^{(2-p(x)) \frac{p(x)}{2}} d x \\
& \leq \int_{V_{p(x)}}\left(A^{(1)}\left(\Delta u_{n}, \Delta u\right)\right)^{\frac{p(x)}{2}}\left(\int_{V_{p(x)}} A^{(1)}\left(\Delta u_{n}, \Delta u\right) d x\right)^{-\frac{p(x)}{2}}\left(C^{(1)}\left(\Delta u_{n}, \Delta u\right)\right)^{(2-p(x))^{\frac{p(x)}{2}}} d x \\
& \leq \int_{V_{p(x)}}\left[A^{(1)}\left(\Delta u_{n}, \Delta u\right)\left(\int_{V_{p(x)}} A^{(1)}\left(\Delta u_{n}, \Delta u\right) d x\right)^{-\frac{1}{2}}+\left(C^{(1)}\left(\Delta u_{n}, \Delta u\right)\right)^{p(x)}\right] d x \\
& \leq 1+\int_{\Omega}\left(C^{(1)}\left(\Delta u_{n}, \Delta u\right)\right)^{p(x)} d x .
\end{aligned}
$$

Hence, by relation 25,

$$
\frac{1}{c_{4}} \int_{V_{p(x)}}\left|\Delta u_{n}-\Delta u\right|^{p(x)} d x \leq\left(\int_{V_{p(x)}} A^{(1)}\left(\Delta u_{n}, \Delta u\right) d x\right)^{\frac{1}{2}}\left[1+\int_{\Omega}\left(C^{(1)}\left(\Delta u_{n}, \Delta u\right)\right)^{p(x)} d x\right] .
$$


We also have

$$
\frac{1}{c_{4}} \int_{V_{p(x)}}\left|\nabla u_{n}-\nabla u\right|^{p(x)} d x \leq\left(\int_{V_{p(x)}} A^{(N)}\left(\nabla u_{n}, \nabla u\right) d x\right)^{\frac{1}{2}}\left[1+\int_{\Omega}\left(C^{(N)}\left(\nabla u_{n}, \nabla u\right)\right)^{p(x)} d x\right] .
$$

By (23), 225), (27) and (28), we have

$$
\int_{\Omega}\left|\Delta u_{n}-\Delta u\right|^{p(x)} d x=\int_{U_{p(x)}}\left|\Delta u_{n}-\Delta u\right|^{p(x)} d x+\int_{V_{p(x)}}\left|\Delta u_{n}-\Delta u\right|^{p(x)} d x \rightarrow 0
$$

when $n \rightarrow \infty$. Similarly, from (24), (26), (27) and (29) we have

$$
\int_{\Omega}\left|\nabla u_{n}-\nabla u\right|^{p(x)} d x=\int_{U_{p(x)}}\left|\nabla u_{n}-\nabla u\right|^{p(x)} d x+\int_{V_{p(x)}}\left|\nabla u_{n}-\nabla u\right|^{p(x)} d x \rightarrow 0 .
$$

Therefore,

$$
\left\|u_{n}-u\right\|^{p^{+}} \leq \int_{\Omega}\left(\left|\Delta u_{n}-\Delta u\right|^{p(x)}+\left|\nabla u_{n}-\nabla u\right|^{p(x)}\right) d x \rightarrow 0
$$

when $n \rightarrow \infty$, we deduce that $\left\{u_{n}\right\}$ converges strongly to $u$ in $X$. Since $J_{\lambda} \in C^{1}(X, \mathbb{R})$, we conclude that

$$
J_{\lambda}^{\prime}\left(u_{n}\right) \rightarrow J_{\lambda}^{\prime}(u), \text { as } n \rightarrow \infty
$$

Relations (15) and (32) show that $J_{\lambda}^{\prime}(u)=0$ and thus $u$ is a weak solution for problem (1). Moreover, by relation (32), it follows that $J_{\lambda}(u)<0$ and thus, $u$ is a nontrivial weak solution for (1). The proof of Theorem 2.5 is complete.

\section{Acknowledgments}

The author would like to thank the referees for their suggestions and helpful comments which improved the presentation of the original manuscript.

\section{References}

[1] G.A. Afrouzi, M. Mirzapour, N.T. Chung, Existence and multiplicity of solutions for Kirchhoff type problems involving $p(x)$-biharmonic operators, Z. Anal. Anwend., 33 (2014), 289-303.

[2] K.B. Ali, A. Ghanmi, K. Kefi, Minimax method involving singular $p(x)$-Kirchhoff equation, J. Math. Physics, 58 (2017): 111505.

[3] H. Ansari, S.M. Vaezpour, Existence and multiplicity of solutions for fourth-order elliptic Kirchhoff equations with potential term, Complex Var. Elliptic Equ., 60 (2015), 668-695.

[4] A. Ayoujil, A.R. El Amrouss, On the spectrum of a fourth order elliptic equation with variable exponent, Nonlinear Anal., 71 (2009), 4916-4926.

[5] J.M. Ball, Initial-boundary value for an extensible beam, J. Math. Anal. Appl., 42 (1973), 61-90.

[6] G. Bonanno, A. Chinni, Existence and multiplicity of weak solutions for elliptic Dirichlet problems with variable exponent, J. Math. Anal. Appl., 418 (2014), 812-827.

[7] M.M. Boureanu, V. Radulescu, D. Repovs, On a (.)-biharmonic problem with no-flux boundary condition, Comput. \& Math. Appl., 72 (2016), 2505-2515.

[8] A. Cabada, G.M. Figueiredo, A generalization of an extensible beam equation with critical growth in $\mathbb{R}^{N}$, Nonlinear Anal. Real World Appl., 20 (2014), 134-142.

[9] N.T Chung, Q.A. Ngo, Multiple solutions for a class of quasilinear elliptic equations of $p(x)$-Laplacian type with nonlinear boundary conditions, Proc. Royal Soc. Edinburgh Sect. A: Mathematics, 140(2) (2010), 259-272.

[10] N.T. Chung, Multiple solutions for a $p(x)$-Kirchhoff-type equation with sign-changing nonlinearities, Complex Var. Elliptic Equ., 58(12) (2013), 1637-1646.

[11] N.T Chung, Existence of solutions for perturbed fourth order elliptic equations with variable exponents, Electron. J. Qual. Theory Differ. Equ., 2018(96) (2018), 1-19.

[12] N.T Chung, Multiple solutions for a fourth order elliptic equation of Kirchhoff type with variable exponent, Asian-European J. Math., DOI: 10.1142/S1793557120500965. 
[13] F. Colasuonno, P. Pucci, Multiplicity of solutions for $p(x)$-polyharmonic Kirchhoff equations, Nonlinear Anal., 74 (2011), 5962-5974.

[14] G. Dai, Three solutions for a nonlocal Dirichlet boundary value problem involving the $p(x)$-Laplacian, Appl. Anal., 92 (2013), 191-210.

[15] L. Ding, L. Li, Two nontrivial solutions for the nonhomogenous fourth order Kirchhoff equation, Z. Anal. Anwend., 36 (2017), 191-207.

[16] L. Diening, P. Harjulehto, P. Hasto, M. Ruzicka, Lebesgue and Sobolev spaces with variable exponents, Lecture Notes, vol. 2017, Springer-Verlag, Berlin, 2011.

[17] D.E. Edmunds, J. Rákosník, Sobolev embedding with variable exponent, Studia Math., 143 (2000), $267-293$.

[18] I. Ekeland, On the variational principle, J. Math. Anal. Appl., 47 (1974), 324-353.

[19] M. Ferrara, S. Khademloo, S. Heidarkhani, Multiplicity results for perturbed fourth-order Kirchhoff type elliptic problems, Appl. Math. Comput., 234 (2014), 316-325.

[20] A. Ghanmi, Nontrivial solutions for Kirchhoff-type problems involving the $p(x)$-Laplace operator, Rocky Mountain J. Math., 48(4) (2018), 1145-1158.

[21] K. Kefi, V.D. Radulescu, On a $p(x)$-biharmonic problem with singular weights, Z. Angew. Math. Phys., 68 (2017): 80.

[22] L. Kong, Eigenvalues for a fourth order elliptic problem, Proc. Amer. Math. Soc., 143 (2015), 249-258.

[23] M. Massar, El M. Hssini, N. Tsouli, M. Talbi, Infinitely many solutions for a fourth-order Kirchhoff type elliptic problem, J. Math. Comput. Sci., 8 (2014), 33-51.

[24] M. Mihailescu, V.D. Radulescu, On a nonhomogeneous quasilinear eigenvalue problem in Sobolev spaces with variable exponent, Proc. Amer. Math. Soc., 135 (2007), 2929-2937.

[25] J. Musielak, Orlicz Spaces and Modular Spaces, Lecture Notes in Mathematics, Vol. 1034, Springer, Berlin, 1983.

[26] M. Ruzicka, Electrorheological Fluids: Modeling and Mathematical Theory, Springer-Verlag, Berlin, 2002.

[27] Y. Song, S. Shi, Multiplicity of solutions for fourth-order elliptic equations of Kirchhoff type with critical exponent, J. Dyn. Control Syst., 23 (2017), 375-386.

[28] F. Wang, M. Avci, Y. An, Existence of solutions for fourth order elliptic equations of Kirchhoff type, J. Math. Anal. Appl., 409 (2014), 140-146.

[29] F. Wang, Y. An, Existence and multiplicity of solutions for a fourth-order elliptic equation, Bound. Value Probl., 2012 (2012): 6 .

[30] A. Zang, Y. Fu, Interpolation inequalities for derivatives in variable exponent Lebesgue-Sobolev spaces, Nonlinear Anal., 69 (2008), 3629-3636.

[31] V.V. Zhikov, Averaging of functionals of the calculus of variations and elasticity theory, Math. USSR. Izv., 9 (1987), 33-66. 